\title{
Automating prestack migration analysis using common focal point gathers
}

\author{
Scott A. Morton*, Cray Research, Inc; Jan Thorbecke, Delft University of Technology
}

\section{Summary}

We have implemented the common focal point (CFP) imaging technique and have developed a method for automating iterative 3-D prestack migration analysis using CFP gathers to update travel-time operators.

\section{Introduction}

Prestack depth migration has proven to be a useful imaging method and has become a part of mainstream seismic technology. However, the utility of prestack depth migration is fundamentally limited by our ability to determine models of the earth accurately, quickly and efficiently.

CFP technology (Berkhout, 1996a,b) addresses these limitations in a novel way. By breaking the model determination process into two simpler, more easily solved problems, those of operator estimation and macro model estimation, the final solution will typically be more accurate. Moreover, by providing rapid local convergence to the correct travel-time operators, migration analysis can be automated, resulting in reduced manpower requirements and shorter time-to-solution.

In this paper, we briefly review some aspects of CFP technology and present results of using CFP gathers for automating the iterative process of 3-D prestack depth migration analysis.

\section{Method}

Our approach to automated prestack analysis starts from initial estimates of the travel-time operators, which could be calculated from a previously estimated model, derived from stacking velocities, or even taken from a regional trend. Then the interative process of alternating between generating a CFP gather and updating the operator from the gather proceeds until the convergence criteria are satisfied.

Since detailed mathematics for CFP technology has been presented elsewhere (Berkhout and Rietveld, 1995; Berkhout, 1996a,b), we present only a simple, conceptual description of the generation of a CFP gather and the update of the operator. For a specific position in the image volume, $\mathbf{x}$, a CFP gather is calculated by focusing the input traces in detection; a simple Kirchhoff implementation looks like:

$\operatorname{CFP}\left(\mathbf{x}_{\mathbf{s}}, \tau ; \mathbf{x}\right)=\int d^{2} \mathbf{x}_{\mathbf{r}} I\left(\mathbf{x}_{\mathbf{s}}, \mathbf{x}_{\mathbf{r}}, t=\tau+T\left(\mathbf{x}, \mathbf{x}_{\mathbf{r}}\right)\right)$ where $\mathbf{x}_{\mathbf{S}}$ and $\mathbf{x}_{\mathbf{r}}$ are the source and receiver surface locations, respectively, for an input trace $I\left(\mathbf{x}_{\mathbf{s}}, \mathbf{x}_{\mathbf{r}}, t\right)$ and $T\left(\mathbf{x}, \mathbf{x}_{\mathbf{r}}\right)$ is the travel time from the focal point $\mathbf{x}$ to the receiver. Although in general an amplitude factor should be included, we neglect it here since our interest is solely in the kinematics.

If the correct travel times have been used, the response of the focal point $\mathbf{x}$ in the CFP domain is kinematically identical to $T\left(\mathbf{x}, \mathbf{x}_{\mathbf{s}}\right)$; summing along $T\left(\mathbf{x}, \mathbf{x}_{\mathbf{s}}\right)$ then yields the Kirchhoff image at $\mathbf{x}$. However, any error in the travel times used to calculate the gather shows up in a mismatch between the CFP response and the approximate travel times. Fortunately, a small error in the travel times is matched by a corresponding opposite error on the CFP gather, with the correct travel time lying approximately halfway between the two (Berkhout, 1996a,b). This allows us to calculate only a small window of a CFP gather around the travel-time operator (i.e., for times $\tau$ near $\left.T\left(\mathbf{x}, \mathbf{x}_{\mathbf{s}}\right)\right)$, dramatically reducing the required computation. We then automatically generate an updated operator by picking and tracking an event on the CFP gather and averaging it with the operator used to construct the gather. When the CFP response matches the operator, the "windowed" CFP gather is flat (at zero "differential time") and the process has converged.

The simplicity of this approach results from the fact that the operator and the CFP gather are both functions of the same independent variables, the surface location and the image point. In addition, these provide a natural domain for prestack data, allowing CFP technology fuller access to the information present in the data. In contrast, most current methods of prestack analysis involve iterating between common-offset images and the underlying velocity model, where the relationship is much less direct.

A key component of applying our iterated approach to prestack migration analysis in an automated fashion is the technology to pick and track events on the CFP gathers. For the results in this abstract, we used a very simple method; we picked the maximum event on the stack of the CFP gather and tracked it by following the envelopes of events from trace-to-trace within the gather.

\section{Examples}

We first illustrate our approach by presenting results from a 3 -D constant $(2000 \mathrm{~m} / \mathrm{s})$ velocity model containing a flat reflector at a depth of $800 \mathrm{~m}$. We generated syn- 


\section{Automating prestack analysis}

thetic data consisting of seven 3000-m seismic lines separated by $500 \mathrm{~m}$ with shot and receiver spacing of $25 \mathrm{~m}$. We then ran a series of experiments by starting the automated scheme with constant-velocity travel times of the form $T(L)=\sqrt{t_{0}^{2}+L^{2} / v_{0}^{2}}$, where $L$ is the surface distance between the focal (or image) point and the source or receiver, and $t_{0}$ and $v_{0}$ are a vertical time and velocity, respectively. Figure 2 presents the results of these experiments; each plot is the collection of "windowed" CFP gathers, which we will refer to as "differential time shift" (DTS) panels, from the iterations of a single experiment and is labeled by its corresponding $t_{0}$ and $v_{0}$.

All these cases converged within a few iterations and resulted in DTS panels which were flat at $t=0$ to within a single time sample (4 ms). We compared the resulting travel-time operators to the correct operator for the constant velocity model and found our results to be accurate to within $2 \mathrm{~ms}$ and $0.5 \%$ along the center lines. Note that because of the 2-D nature of the data geometry there isn't useful signal off this line in our CFP gathers, clearly indicating the role acquisition can play in limiting prestack analysis.

We also tested our method on a $2500 \mathrm{~m}$ by $2500 \mathrm{~m}$ region of the SEG/EAGE overthrust model. The input data consisted of a 9 by 9 grid of shot records, each shot record containing a 100 by 100 array of receivers. We located our focal point at the $(x=4075, y=3750)$ surface position and started our automated scheme with an initial constantvelocity travel-time operator using a velocity of $3000 \mathrm{~m} / \mathrm{s}$ and a vertical time of $940 \mathrm{~ms}$. The vertical time was chosen from the zero-offset data for the interface whose depth varies between $2800 \mathrm{~m}$ at $x=2500$ and $2300 \mathrm{~m}$ at $x=5000$; see Figure 1, while the velocity was chosen intentionally low. The results of the experiment are displayed in Figure 3, where again we found that the process converged and the resulting DTS panels were flat to within a single time sample.

\section{Conclusions}

Using very simple technology to automate the updating of travel-time operators, we were able to successfully flatten the windowed CFP gathers (i.e., the DTS panels) in just a few iterations, indicating convergence to the correct travel times. This kind of approach holds great promise for automating prestack migration analysis and, when combined with tomographic inversion of the resulting travel times, may ultimately result in a fully automated method for imaging and model determination.

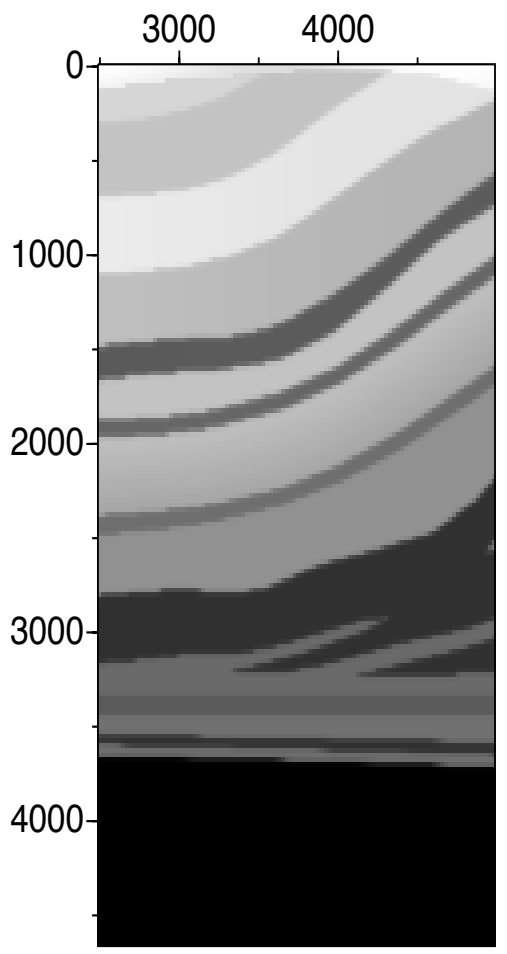

Fig. 1: A constant $y=3750 \mathrm{~m}$ slice throught the middle of the SEG/EAGE overthrust model. The velocity varies from about $2500 \mathrm{~m} / \mathrm{s}$ near the surface to $6000 \mathrm{~m} / \mathrm{s}$ at the bottom of the model. The DTS panels in Figure 3 were generated for the $x=4075 \mathrm{~m}$ position in this slice.

\section{Acknowledgements}

We would like to thank Sandia National Laboratory for providing us with their data for the SEG/EAGE overthrust model. SM would particularly like to thank Prof. Berkhout for suggesting and encouraging a 3-D implementation of the CFP technology.

\section{References}

Berkhout, A.J., and Rietveld, W.E.A., 1995, Prestack migration in terms of double dynamic focusing, 65th Ann. Int. Mtg. SEG, Exp. Abstracts.

Berkhout, A.J., 1996a, Pushing the limits of seismic imaging - Part I. Prestack migration in terms of double dynamic focusing, Geophysics, in press.

Berkhout, A.J., 1996b, Pushing the limits of seismic imaging - Part II. Integration of prestack migration, velocity estimation and AVO analysis, Geophysics, in press. 
Automating prestack analysis
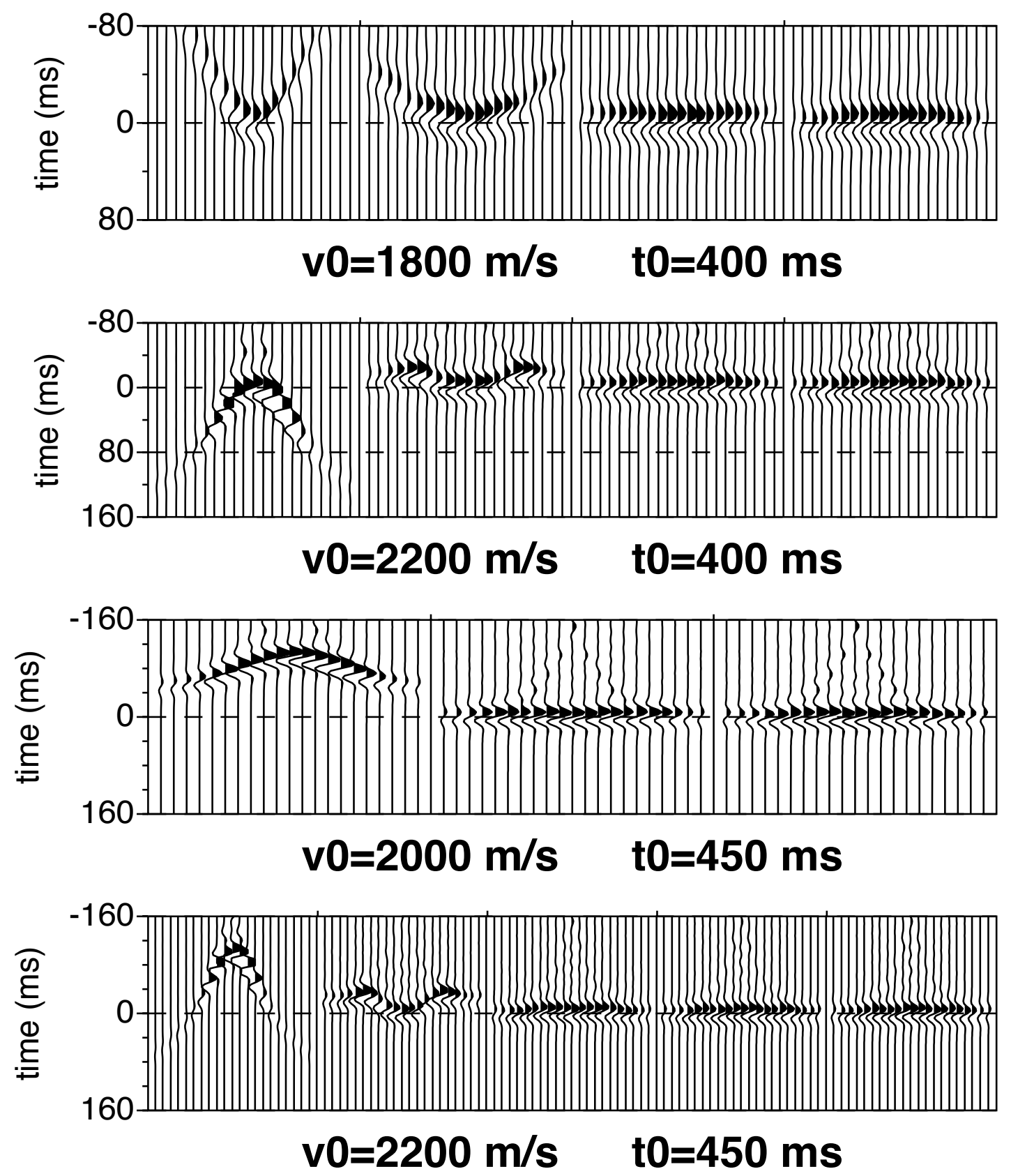

Fig. 2: DTS panels for a flat reflector in a constant velocity medium. Each plot contains the collection of DTS panels from a single experiment, where the initial travel-time operators were constructed using the velocities and vertical times indicated below the plot. The correct velocity and vertical time for the model is $2000 \mathrm{~m} / \mathrm{s}$ and $400 \mathrm{~ms}$, respectively. The DTS panels consist of 21 traces each, are separated by a blank trace and tick marks, and correspond to the center inline slice of the full 3-D CFP gather. Iteration 0 is at the left (the gather from the initial travel-time operator), with increasing iteration number toward the right. Although the experiments differed in the number of iterations required, in all cases the final DTS panels are flat to within the time discretization (4 ms) and the travel-time operators are within $2 \mathrm{~ms}$ of the analytical answer, indicating convergence. 
Automating prestack analysis
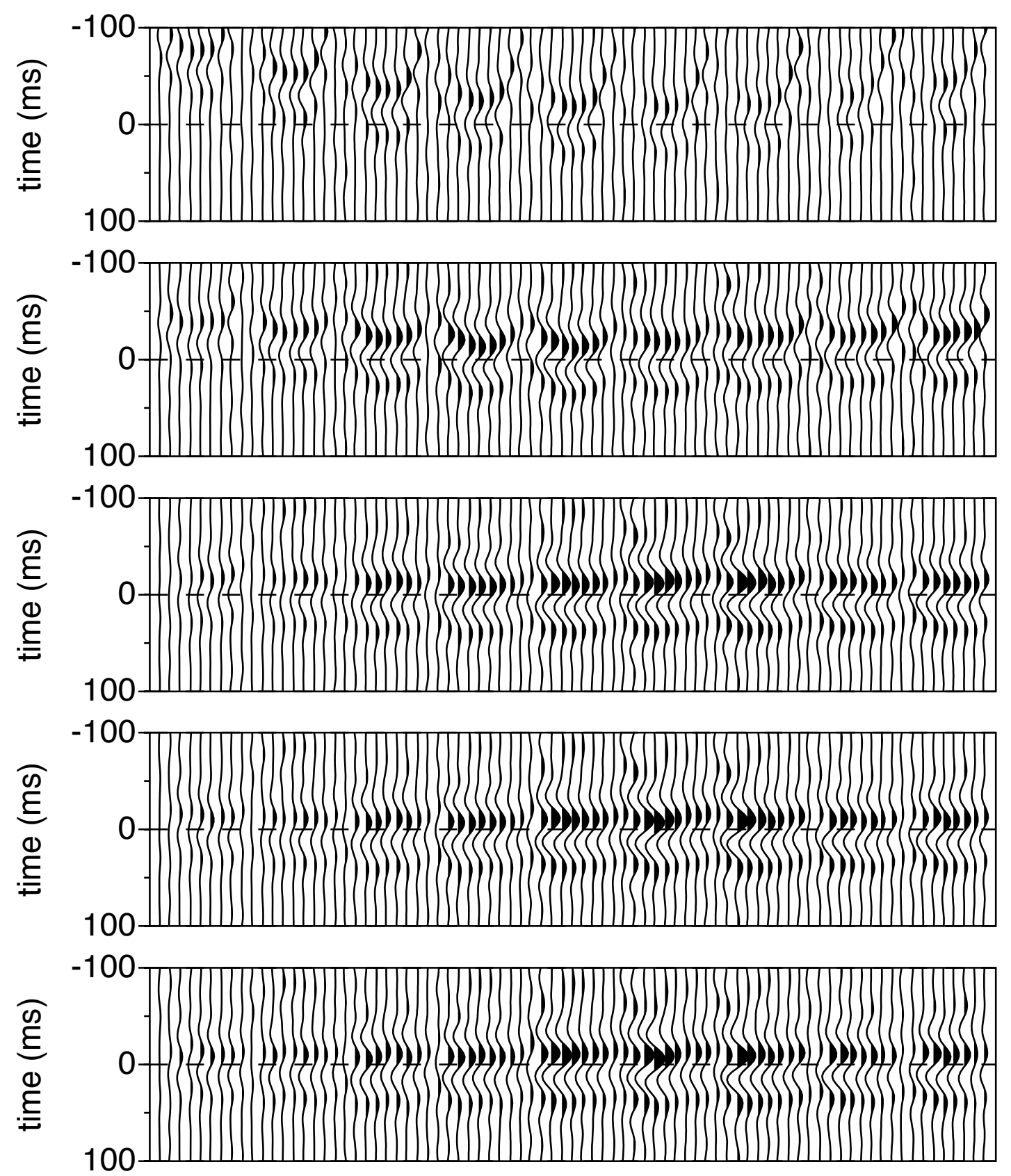

Fig. 3: DTS panels for a location near the middle of the portion of the SEG/EAGE overthrust model in Figure 1. Each plot is a 3-D DTS panel, consisting of a 9 by 9 grid of traces. The traces are displayed as constant y-slices with $x$ varying first. The top panel is from the initial travel-time operator. By the 4th iteration (bottom), the amplitudes have grown dramatically and the DTS panel is flat at $t=0$ to within the time discretization $(4 \mathrm{~ms})$. 